\title{
Self-adapting root-MUSIC algorithm and its real-valued formulation for acoustic vector sensor array
}

\author{
Peng Wang ${ }^{1,3}$, Guo-jun Zhang ${ }^{1,2}$, Chen-yang Xue ${ }^{1,2}$, Wen-dong Zhang ${ }^{1,2}$ and Ji-jun Xiong ${ }^{1,2^{*}}$
}

\begin{abstract}
In this paper, based on the root-MUSIC algorithm for acoustic pressure sensor array, a new self-adapting root-MUSIC algorithm for acoustic vector sensor array is proposed by self-adaptive selecting the lead orientation vector, and its real-valued formulation by Forward-Backward(FB) smoothing and real-valued inverse covariance matrix is also proposed, which can reduce the computational complexity and distinguish the coherent signals. The simulation experiment results show the better performance of two new algorithm with low Signal-to-Noise (SNR) in direction of arrival (DOA) estimation than traditional MUSIC algorithm, and the experiment results using MEMS vector hydrophone array in lake trails show the engineering practicability of two new algorithms.
\end{abstract}

Keywords: Acoustic vector sensor, DOA, MUSIC

\section{Background}

Compared to traditional acoustic pressure sensor, the acoustic vector sensor can measure both the scalar acoustic pressure and the acoustic particle velocity vector at a certain point of the acoustic field. So it possesses higher direction sensitivity and can acquire more measurement information [1-3]. By taking advantage of the extra information, vector sensors arrays are able to improve the direction-of-arrival (DOA) estimation performance without increasing array aperture size. Nehorai and Paldi have developed the measurement model of the acoustic vector sensor array for dealing with narrowband sources [4], many methods such as MUSIC algorithms have been proposed for applying acoustic vector sensor array to DOA estimation problems [5-8].

Root-MUSIC algorithm is a polynomial form of MUSIC algorithm $[7,8]$. This algorithm adopts the roots of a polynomial to replace the search for spatial spectrum in MUSIC algorithm, reducing the calculation amount

\footnotetext{
* Correspondence: wpncit@163.com

${ }^{1}$ Key Laboratory of Instrumentation Science \& Dynamic Measurement, North University of China, Taiyuan 030051, China

${ }^{2}$ Science and Technology on Electronic Test \& Measurement Laboratory,

North University of China, Taiyuan 030051, China

Full list of author information is available at the end of the article
}

and improving estimation performance. Nevertheless, it is mainly applied to acoustic pressure sensor array.

Combining the Micro Electronic Mechanical Systems (MEMS) technology with design of vector hydrophone, it can break the performance limitation of existing hydrophone. A novel biomimetic MEMS vector hydrophone has been developed by Xue and co-authous (Figure 1), and has been measured for index [9-12].

In this paper, a self-adapting root-MUSIC algorithm and its real-valued formulation for acoustic vector sensor array are proposed. Furthermore, the comparison of performance between this algorithm and MUSIC algorithm has been made by simulation method. Finally, the engineering practicability has been tested according to the experimental data of MEMS vector hydrophone array in lake trials.

\section{Signal model of acoustic vector sensor array}

Consider $\mathrm{N}$ far-field narrowband signals incident on an uniform line array of $M$ acoustic vector sensors along the $x$-axis in space, from directions $\boldsymbol{\theta}=\left[\theta_{1}, \theta_{2}, \cdots, \theta_{N}\right]^{T}$, the received signal vector of the array can be expressed as

$$
\mathbf{Z}(t)=\mathbf{A}(\boldsymbol{\theta}) \mathbf{S}(t)+\mathbf{N}_{v}(t),
$$

where $\mathbf{Z}(t)$ is the $3 M \times 1$ snapshot data vector of the array, $\mathbf{S}(t)$ is the $N \times 1$ vector of the signal, $\mathbf{N}_{v}(t)$ is the 


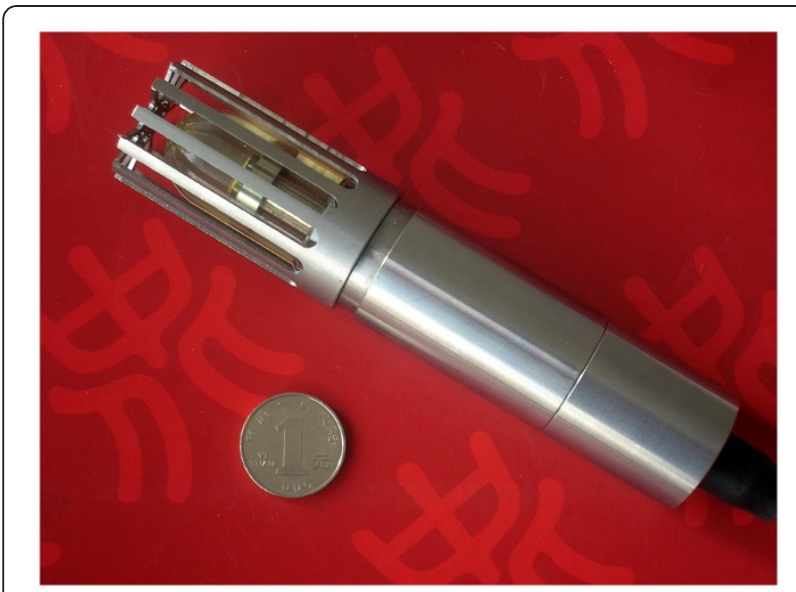

Figure 1 MEMS vector hydrophone.

$3 M \times 1$ vector of the Gaussian noise data vector, and the noise and the signal are independent, $\mathbf{A}(\boldsymbol{\theta})$ is the steering vector matrix of the acoustic vector sensor array.

$$
\begin{aligned}
& \mathbf{A}(\boldsymbol{\theta})=\left[\mathbf{a}\left(\theta_{1}\right), \mathbf{a}\left(\theta_{2}\right), \cdots, \mathbf{a}\left(\theta_{N}\right)\right] \\
& \quad=\left[\mathbf{a}_{1}\left(\theta_{1}\right) \otimes \mathbf{u}_{1}, \mathbf{a}_{2}\left(\theta_{2}\right) \otimes \mathbf{u}_{2}, \cdots, \mathbf{a}_{N}\left(\theta_{N}\right) \otimes \mathbf{u}_{N}\right]
\end{aligned}
$$

where $\mathrm{a}_{k}\left(\theta_{k}\right)=\left[1, \mathrm{e}^{-j \beta_{k}}, \mathrm{e}^{-j 2 \beta_{k}}, \cdots, \mathrm{e}^{-j(M-1)_{k}}\right]^{T} \quad$ is the acoustic pressure corresponding of the $k$ th signal, $\beta_{k}=$ $\frac{2 \pi}{\lambda} d \sin \theta_{k}$, in which $d$ is the inter-element spacing, and $\lambda$ is the wavelength corresponding to the maximum frequency of signals. $\mathbf{u}_{k}=\left[1, \cos \theta_{k}, \sin \theta_{k}\right]^{T}$ is the direction vector of the $k$ th signal, and the notation $\otimes$ denotes the Kronecker product.

So the covariance matrix for the array received signal is given by

$$
\begin{aligned}
\mathbf{R} & =E\left[\mathbf{Z}(t) \mathbf{Z}^{H}(t)\right] \\
& =\mathbf{A} E\left[\mathbf{S}(t) \mathbf{S}^{H}(t)\right] \mathbf{A}^{H}+E\left[\mathbf{N}(t) \mathbf{N}^{H}(t)\right] \\
& =\mathbf{A} \mathbf{R}_{S} \mathbf{A}^{H}+\sigma^{2} \mathbf{I}
\end{aligned}
$$

where $\mathbf{R}_{S}$ is the signal covariance matrix, $\sigma^{2}$ is the energy of Gaussian white noise, $\mathbf{I}$ is the normalized noise covariance matrix, and $(\cdot)^{H}$ stands for complex conjugate transpose.

From the theory of subspace decomposition, the eigendecomposition is

$$
\mathbf{R}=\mathbf{U}_{S} \boldsymbol{\Sigma}_{S} \mathbf{U}_{S}^{H}+\mathbf{U}_{N} \boldsymbol{\Sigma}_{N} \mathbf{U}_{N}^{H}
$$

where $\mathbf{U}_{S}$ is the signal subspace spanned by eigenvectors corresponding to major eigenvalues of matrix $\mathbf{R}, \mathbf{U}_{N}$ is the noise subspace spanned by eigenvectors corresponding to small eigenvalues of matrix $\mathbf{R}$.
In practical calculation, the received data are finite, so the covariance matrix $\mathbf{R}$ can be estimated as

$$
\hat{\mathbf{R}}=\frac{1}{L} \sum_{i=1}^{L} \mathbf{Z}(t) \mathbf{Z}^{H}(t)
$$

where $L$ is the number of snapshots.

\section{Self-adapting root-MUSIC algorithm for vector sensor array}

The basic idea of self-adapting root-MUSIC algorithm is: firstly weight summation for three-way signal of vector sensor, select the self-adaptive lead orientation, then construct polynomial by noise subspace, and finally estimate DOA of signals by finding the roots of polynomial.

\section{Selection of lead orientation vector}

Weight $1, \cos \phi, \sin \phi$ to the output signal $p_{i}(t), v_{i x}(t)$, $v_{i y}(t)$ of $i$ th vector sensor respectively, and make sum

$$
y_{i}(t)=p_{i}(t)+v_{i x}(t) \cos \phi+v_{i y}(t) \sin \phi,
$$

then the average power is $\mathrm{P}_{i}(\phi)=\mathrm{E}\left[\left|y_{i}(t)\right|^{2}\right]$.

The function of weight corresponds to make electronic rotary for the output of the vector sensor, the direction $\phi$ which reflects the maximum energy is the signal direction [13].

$\mathrm{P}_{i}(\phi)$ is the output of spatial spectrum of $i$ th vector sensor with $\phi$ relevant, reflects the energy distribution in space. It is the equivalent of a spatial filter, and can implement the signal and noise separation based on the orientation difference of the signal and interference.

The vector form of (6) for vector sensor array can be written as

$$
\mathbf{Y}=\mathbf{W} \cdot \mathbf{Z}
$$

where $\mathbf{W}=\operatorname{diag}[1, \cos \phi, \sin \phi, \cdots, 1, \cos \phi, \sin \phi]$.

Take

$$
P(\phi)=\frac{1}{M} \sum_{i=1}^{M} P_{i}(\phi),
$$

where $\mathrm{P}(\phi)$ is spatial spectrum of array. The lead orientation $\phi_{0}$ can be obtained from the maximum of $\mathrm{P}(\phi)$ for $\phi \in[0,2 \pi]$.

The lead orientation vector can be received as

$$
\mathbf{u}=\left[1, \cos \phi_{0}, \sin \phi_{0}\right]^{T}
$$

where $\mathbf{u}$ is also known as self-adaptive lead vector. 


\section{Construction of the polynomial}

Define the polynomial

$$
f(z)=z^{M-1} \mathbf{F}^{T}(1 / z) \mathbf{U}_{N} \mathbf{U}_{N}^{H} \mathbf{F}(z),
$$

where $\mathbf{F}(z)=\left[1, z, \cdots, z^{M-1}\right]^{T} \otimes \mathbf{u}, z=\exp (j \beta), \beta=(2 \pi / \lambda) d$ $\sin \theta$, and $\theta$ is the azimuth angle of the signals to be estimated.

Let

$$
\mathbf{B}=\left(\begin{array}{cccc}
\mathbf{b}_{11} & \mathbf{b}_{12} & \cdots & \mathbf{b}_{1 M} \\
\mathbf{b}_{21} & \mathbf{b}_{22} & \cdots & \mathbf{b}_{2 M} \\
\vdots & \vdots & \ddots & \vdots \\
\mathbf{b}_{M 1} & \mathbf{b}_{M 2} & \cdots & \mathbf{b}_{M M}
\end{array}\right)=\mathbf{U}_{N} \mathbf{U}_{N}^{H}
$$

where $\mathbf{b}_{i j}(i, j=1,2, \cdots, M)$ are $3 \times 3$ symmetry submatrix.

Then

$$
\begin{aligned}
f(z)= & z^{M-1} \mathbf{F}^{T}(1 / z) \mathbf{B F}(z)=\mathbf{u} \mathbf{b}_{M 1} \mathbf{u}^{H} \\
& +z \mathbf{u} \sum_{i=1}^{2} \mathbf{b}_{i+M-2, i} \mathbf{u}^{T}+\cdots+z^{M-1} \mathbf{u} \sum_{i=1}^{M} \mathbf{b}_{i, i} \mathbf{u}^{T} \\
& +z^{M} \mathbf{u} \sum_{i=1}^{M-1} \mathbf{b}_{i, i+1} \mathbf{u}^{T}+\cdots+z^{2 M-3} \mathbf{u} \sum_{i=1}^{2} \mathbf{b}_{i, i+M-2} \mathbf{u}^{T} \\
& +z^{2 M-2} \mathbf{u} \mathbf{b}_{1 M} \mathbf{u}^{T}=\sum_{k=1}^{M}\left(\mathbf{u} \sum_{i=1}^{k} \mathbf{b}_{i+M-k, i} \mathbf{u}^{T}\right) z^{k-1} \\
& +\sum_{k=1}^{M-1}\left(\mathbf{u} \sum_{i=1}^{M-k} \mathbf{b}_{i, i+k} \mathbf{u}^{T}\right) z^{M+k-1},
\end{aligned}
$$

So the order of the polynomial $f(z)$ is $2(M-1)$, it has $(M-1)$ pair roots which every two conjugate with each another. and there are $N$ roots which lie on the unit circle,

$$
z_{i}=\exp \left(j \beta_{i}\right), i=1,2, \cdots, N \text {. }
$$

In practical calculation, considering the error of covariance matrix, the $N$ roots $\hat{z}_{i}$ nearest to the unit circle can be estimated as the DOAs of the signals.

$$
\hat{\theta}_{i}=\arcsin \left(\frac{\lambda}{2 \pi d} \arg \left\{\hat{z}_{i}\right\}\right), i=1,2, \cdots, N .
$$

To sum up, the self-adapting root-MUSIC algorithm can be formulated as the following six-step procedure: (5).

Step 1: Compute $\mathbf{R}$ by (3), and the estimate is given by

Step 2: Obtain $\mathbf{U}_{N}$ from the eigendecomposition of $\mathbf{R}$ by (4).

Step 3: Compute the lead vector $\mathbf{u}$ by (8).

Step 4: Construct the polynomial $f(z)$ by (11).

Step 5: Find the root of the polynomial $f(z)$, and select the roots $\hat{z}_{i}$ that are nearest to the unit circle as being the roots corresponding to the DOA estimates.

Step 6: Receive $\hat{\theta}_{i}$ to the DOA estimates by (12).

\section{RV-Root-MUSIC algorithm}

In the above method, the computational complexity will be reduced greatly if making eigendecomposition for a real-valued matrix instead of complex covariance matrix $\mathbf{R}[14]$. The specific process is as follows:

Define

$$
\mathbf{J}_{3 M}=\mathbf{J}_{M} \otimes \mathbf{I}_{3},
$$

where $\mathbf{J}_{M}$ is the $M \times M$ exchange matrix with ones on its antidiagonal and zeros elsewhere, and $\mathbf{I}_{3}$ is a $3 \times 3$ identity matrix.

Import the Forward-Backward(FB) smoothing matrix $\mathbf{R}_{\mathrm{FB}}$ as[15],

$$
\mathbf{R}_{\mathrm{FB}}=\frac{1}{2}\left(\mathbf{R}+\mathbf{J}_{3 M} \mathbf{R}^{*} \mathbf{J}_{3 M}\right),
$$

where $(\cdot)^{\prime \prime}$ stands for complex conjugate.

The real-valued covariance matrix $\mathbf{C}$ can be obtained by

$$
\mathbf{C}=\mathbf{P}^{H} \mathbf{R}_{\mathrm{FB}} \mathbf{P},
$$

where $\mathbf{P}=\mathbf{Q} \otimes \mathbf{I}_{3}$ is a sparse matrix with real-valued conversion [14], and matrix $\mathbf{Q}$ can be chosen for arrays with an even and odd number of sensors respectively by (16) and (17).

$$
\begin{aligned}
& \mathbf{Q}_{2 n}=\frac{1}{\sqrt{2}}\left(\begin{array}{cc}
\mathbf{I}_{n} & j \mathbf{I}_{n} \\
\mathbf{J}_{n} & -j \mathbf{J}_{n}
\end{array}\right), \\
& \mathbf{Q}_{2 n+1}=\frac{1}{\sqrt{2}}\left(\begin{array}{ccc}
\mathbf{I}_{n} & \mathbf{0} & j \mathbf{I}_{n} \\
\mathbf{0}^{T} & \sqrt{2} & \mathbf{0}^{T} \\
\mathbf{J}_{n} & \mathbf{0} & -j \mathbf{J}_{n}
\end{array}\right),
\end{aligned}
$$

where $\mathbf{0}$ is the $n \times 1$ zero vector.

It is proved that $\mathbf{C}$ is a real-valued covariance matrix as follows.

Because of $\mathbf{Q}^{*}=\mathbf{J Q} \mathrm{JQ}^{*}=\mathbf{Q}$ and $\mathbf{J}^{\mathbf{H}}=\mathbf{J}$, then

$$
\begin{aligned}
\mathbf{P}^{H} \mathbf{J}_{3 M} \mathbf{R}^{*} \mathbf{J}_{3 M} \mathbf{P} & =\left(\mathbf{Q} \otimes \mathbf{I}_{3}\right)^{H}\left(\mathbf{J}_{M} \otimes \mathbf{I}_{3}\right) \mathbf{R}^{*}\left(\mathbf{J}_{M} \otimes \mathbf{I}_{3}\right)\left(\mathbf{Q} \otimes \mathbf{I}_{3}\right) \\
& =\left(\mathbf{Q}^{H} \otimes \mathbf{I}_{3}\right)\left(\mathbf{J}_{M} \otimes \mathbf{I}_{3}\right) \mathbf{R}^{*}\left(\mathbf{J}_{M} \otimes \mathbf{I}_{3}\right)\left(\mathbf{Q} \otimes \mathbf{I}_{3}\right) \\
& =\left[\left(\mathbf{Q}^{H} \mathbf{J}_{M}\right) \otimes \mathbf{I}_{3}\right] \mathbf{R}^{*}\left[\left(\mathbf{J}_{M} \mathbf{Q}\right) \otimes \mathbf{I}_{3}\right] \\
& =\left[\left(\mathbf{Q}^{*}\right)^{H} \otimes \mathbf{I}_{3}\right] \mathbf{R}^{*}\left[\mathbf{Q}^{*} \otimes \mathbf{I}_{3}\right] \\
& =\left[\left(\mathbf{Q} \otimes \mathbf{I}_{3}\right)^{*}\right]^{H} \mathbf{R}^{*}\left[\mathbf{Q} \otimes \mathbf{I}_{3}\right]^{*} \\
& =\left(\mathbf{P}^{*}\right)^{H} \mathbf{R}^{*} \mathbf{P}^{*}=\left(\mathbf{P}^{H} \mathbf{R} \mathbf{P}\right)^{*},
\end{aligned}
$$

So,

$$
\begin{aligned}
\mathbf{C} & =\mathbf{P}^{H} \mathbf{R}_{\mathrm{FB}} \mathbf{P}=\frac{1}{2}\left(\mathbf{P}^{H} \mathbf{R} \mathbf{P}+\mathbf{P}^{H} \mathbf{J}_{3 M} \mathbf{R}^{*} \mathbf{J}_{3 M} \mathbf{P}\right) \\
& =\operatorname{Re}\left(\mathbf{P}^{H} \mathbf{R} \mathbf{P}\right),
\end{aligned}
$$

where $\operatorname{Re}(\cdot)$ is the real part operator. 
Let the eigendecompositions of the matrix $\mathbf{C}$ be defined in a standard way

$$
\mathbf{C}=\mathbf{E}_{S} \boldsymbol{\Lambda}_{S} \mathbf{E}_{S}^{H}+\mathbf{E}_{N} \boldsymbol{\Lambda}_{N} \mathbf{E}_{N}^{H}
$$

Similarly to (9), the real-valued root-MUSIC polynomial can be used

$$
f(z)=z^{M-1} \mathbf{F}^{T}(1 / z) \mathbf{E}_{N} \mathbf{E}_{N}^{H} \mathbf{F}(z)
$$

The computational complexity of self-adapting rootMUSIC algorithm and its real-valued formulation is discussed as follows.

The mainly difference between two methods is that the processing of the covariance matrix. Firstly, the reconstruction of covariance matrix $\mathbf{R}$ by (14) and (15) is necessary for RV-Root-MUSIC algorithm, Since the array covariance matrix $\mathbf{R}$ is a $3 M \times 3 M$ complex matrix, the matrix Ccan be constructed using $2 \cdot(3 M)^{3}$ real multiplications and $(3 M)^{2}(3 M-1)$ real additions by (19).

Secondly, the velocity of convergence for eigendecomposition of the complex matrix $\mathbf{C}$ and the real matrix $\mathbf{R}$ is $O\left(n^{3}\right)$. simultaneously, the noise subspace of the complex matrix $\mathbf{R}$ is also complex, and the noise subspace of the real matrix $\mathbf{C}$ is also real.

Finally, the polynomial $f(z)$ can be constructed via complex matrix $\mathbf{R}$ using $4\left[(3 M)^{2}(3 M-N)+(3 M)^{2}+3 M\right]$ real multiplications and $3\left[(3 M)^{2}(3 M-N-1)+(3 M+1)\right.$ $(3 M-1)]$ real additions by $(9)$, but the polynomial $f(z)$ can be constructed via real matrix $\mathbf{C}$ using $\left[(3 M)^{2}\right.$ $\left.(3 M-N)+(3 M)^{2}+3 M\right]$ real multiplications and $\left[(3 M)^{2}\right.$ $(3 M-N-1)+(3 M+1)(3 M-1)]$ real additions by $(21)$, so it is possible that the computational complexity for real matrix Ccan be reduced up to $75 \%$ real

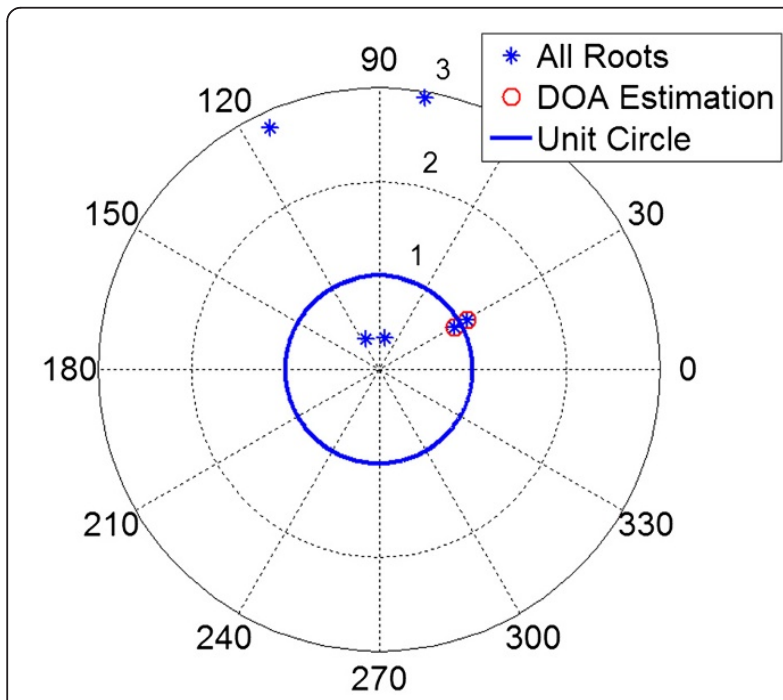

Figure 2 DOA estimation of self-adapting root-MUSIC algorithm.

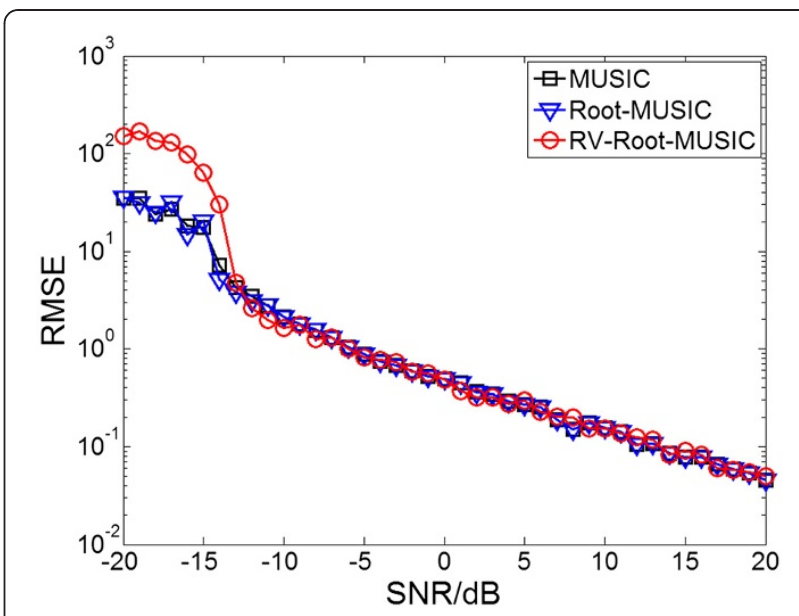

Figure 3 The curve between RMSE and SNR of three methods.

multiplications and $66.7 \%$ real additions compared to the complex matrix $\mathbf{R}$.

From the above analysis, the computational complexity of the RV-Root-MUSIC algorithm is significantly lower than the self-adapting root-MUSIC algorithm thanks to the eigendecomposition of the real-valued matrix $\mathbf{C}$ instead of that of the complex matrix $\mathbf{R}$. On the other hand, due to the inherent forward-backward averaging effect by (14), RV-Root-MUSIC algorithm can separate two completely coherent sources and provides improved estimates for correlated signals. This will be validated in the last experiment of lake trials.

\section{Simulation experiment}

To verify the performance of the proposed self-adapting root-MUSIC algorithm and RV-Root-MUSIC algorithm, simulation experiments are carried out in the following.

The experiment employs the uniform linear array composed of four vector sensors, receives a signal with the frequency being $1 \mathrm{kHz}$ and the angle of incidence being $30^{\circ}$, in which inter-element spacing is half wavelength and the adding noise is Gaussian white noise, and assumes the Signal-to-Noise (SNR) being OdB and the number of snapshots being 200. The DOA estimation using selfadapting root-MUSIC algorithm is shown in Figure 2,

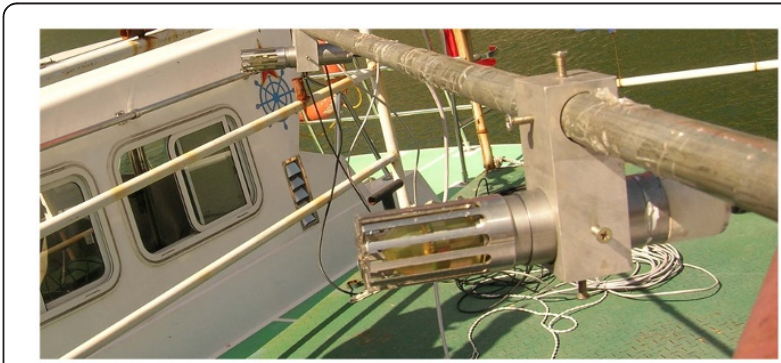

Figure 4 Field experiment. 
Table 1 The DOA estimation result of different frequency signal using two methods

\begin{tabular}{ccc}
\hline $\begin{array}{c}\text { Signal } \\
\text { frequency }\end{array}$ & $\begin{array}{c}\text { Average of DOA } \\
\text { estimation of MUSIC } \\
\text { algorithm( }\left(^{\circ}\right)\end{array}$ & $\begin{array}{c}\text { Average of DOA estimation } \\
\text { of self-adapting root-MUSIC } \\
\text { algorithm( }\left(^{\circ}\right)\end{array}$ \\
\hline $331 \mathrm{~Hz}$ & 90.2768 & 90.0377 \\
$800 \mathrm{~Hz}$ & 89.7857 & 89.8085 \\
$1 \mathrm{kHz}$ & 89.8571 & 89.8598 \\
$1.5 \mathrm{kHz}$ & 89.8636 & 89.4449 \\
$3 \mathrm{kHz}$ & 89.6324 & 89.7719 \\
\hline
\end{tabular}

where the notation "w" stands for all roots of polynomial, "o" for the DOA estimation, and "-" for the unit circle. From Figure 2, it can be seen that the DOA of signal can be correctly estimated using self-adapting root-MUSIC algorithm.

In addition, the performance between the proposed self-adapting root-MUSIC algorithm, the RV-Root-MUSIC algorithm and the traditional MUSIC algorithm is compared. In Figure 3, the root mean square error (RMSE) using 500 independent Monte Carlo trials for each SNR is shown when SNR changes from $-20 \mathrm{~dB}$ to $20 \mathrm{~dB}$. The proposed self-adapting root-MUSIC algorithm and the RV-Root-MUSIC algorithm have identical performance, and they have better performance for low SNRs and almost the same estimation performance for high SNRs with MUSIC algorithm.

Finally, In the above simulation conditions, the statistics for computing time of two algorithms has been made, and it is shown that the integrated computing time of the RV-Root-MUSIC algorithm is average less about $23 \%$ than the self-adapting root-MUSIC algorithm by comparing two methods. Certainly, the computing time of the RV-Root-MUSIC algorithm can be reduced more with the increase of the number of array elements.

\section{Lake trials}

The test experiment has been made in the Fenhe lake (Figure 4). The line array has been composed of two MEMS vector hydrophone with inter-element spacing being $0.5 \mathrm{~m}$, and it has been fixed underwater $10 \mathrm{~m}$ at the side of the ship. The array's compass could take real-time measurement for its pose to keep the array's horizontality. Three experiments have been made respectively.

\section{Experiment 1}

The acoustic emission transducer has been placed in the direction with $90^{\circ}$ of the array, launched $331 \mathrm{~Hz}, 800 \mathrm{~Hz}$, $1 \mathrm{kHz}, 1.5 \mathrm{kHz}, 3 \mathrm{kHz}$ continuous single-frequency signal respectively, the DOA has been estimated for receiving data, once per second. Table 1 is the average result of DOA estimation in different frequency signal using MUSIC algorithm and self-adapting root-MUSIC algorithm, Figure 5 is the time-bearing display of a single-frequency signal with $1.5 \mathrm{kHz}$ using two methods. The result shows the better performance of two methods.

\section{Experiment 2}

The experiment used a motor boat for moving target, which run from about $10^{\circ}$ to about $160^{\circ}$ position, tested track time is about 160s. Broadband noise which motor boat radiate has been narrowband filtered as $800 \mathrm{~Hz}$

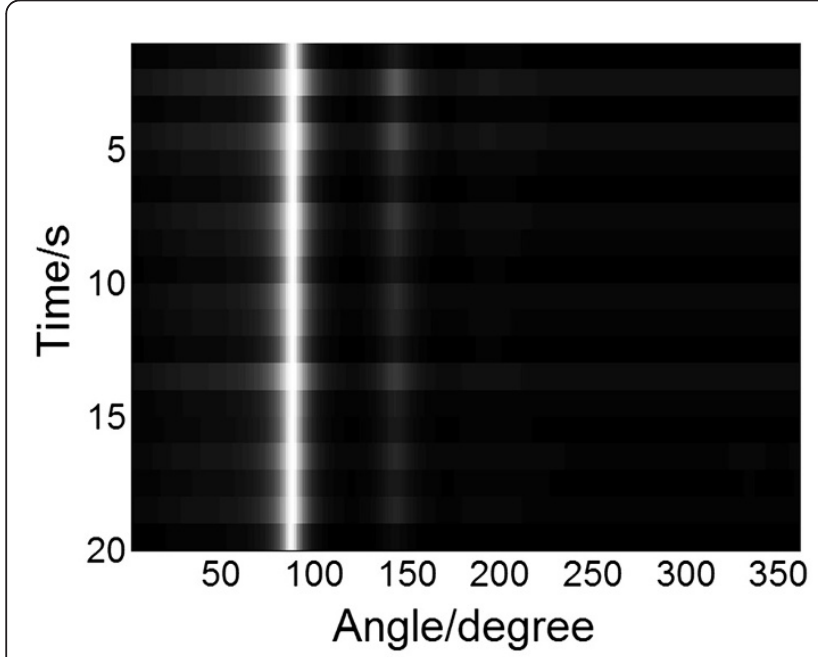

(a) MUSIC algorithm

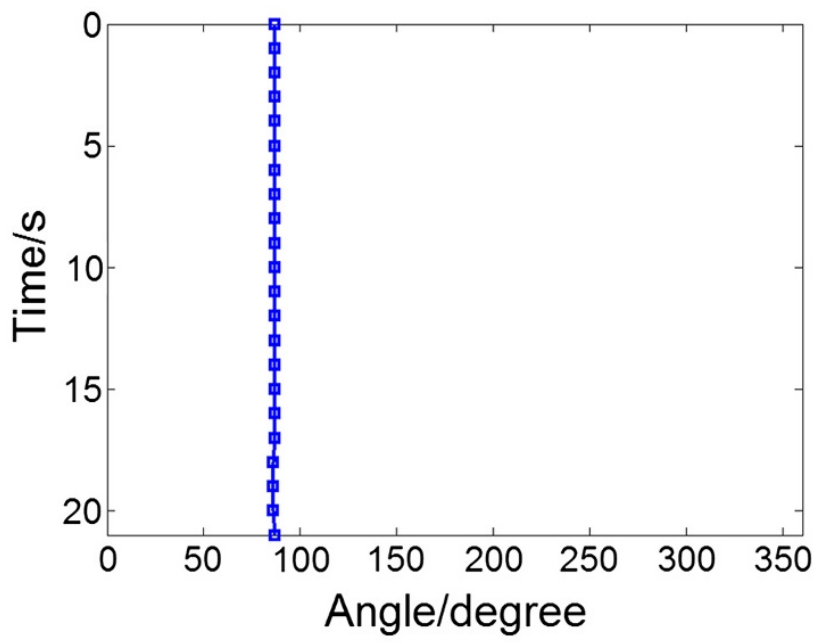

(b) Self-adapting root-MUSIC algorithm

Figure 5 Time-bearing display of signal with $1.5 \mathrm{kHz}$. 
for the center frequency, once per second. Figure 6 shows the time-bearing display of target ship using MUSIC algorithm, conventional beam-forming method (which is also known as Bartlett beam-forming for acoustic vector sensor array) and self-adapting root-MUSIC algorithm respectively, the results are basically consistent with the actual trajectory of motor boat.

\section{Experiment 3}

The experiment used motor boat and emission transducer for two acoustic sources. The acoustic emission transducer has been placed in the direction with $180^{\circ}$ of the array, launched $800 \mathrm{~Hz}$ continuous single-frequency signal, simultaneously, the motor boat run from about $10^{\circ}$ to about $180^{\circ}$ position, tested track time is about 108s. Broadband noise which motor boat radiate has been narrowband filtered as $800 \mathrm{~Hz}$ for the center frequency, once per second.

Here, these two sources can be seen the coherent signals. First the real-valued covariance matrix $\mathbf{C}$ is homologous used to replace the complex matrix $\mathbf{R}$ in MUSIC algorithm and conventional beam-forming method, and then the time-bearing display of two sources using three methods respectively can be seen in Figure 7 . The MUSIC algorithm can be more clearly distinguish between these two sources at the outset, but there will be some ambiguity when two sources approached (Figure 7(a)), and conventional beam-forming method is completely unable to distinguish (Figure 7(b)), but the RV-Root-MUSIC algorithm can clearly distinguish (Figure 7(c)), the results are basically consistent with the actual trajectory of motor boat and emission transducer.

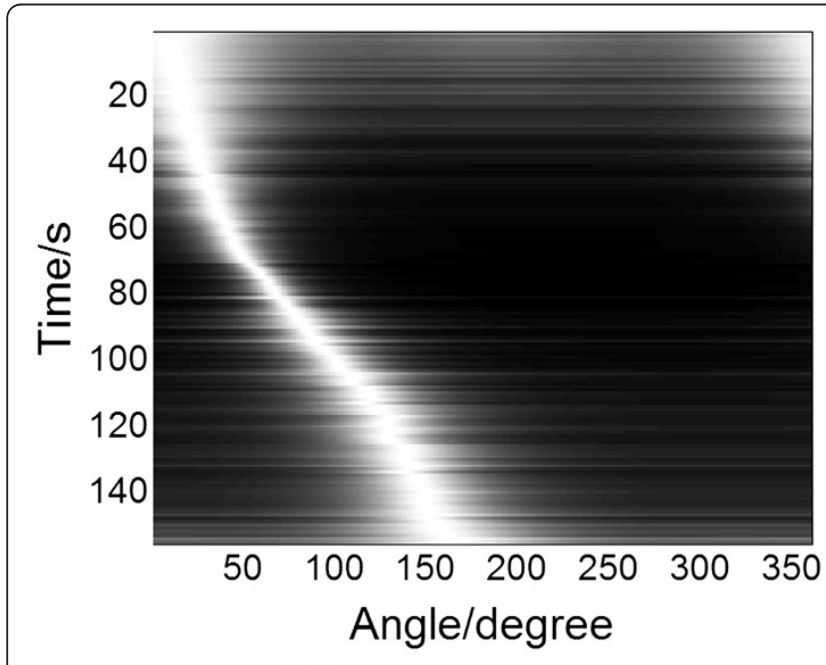

(a) MUSIC algorithm

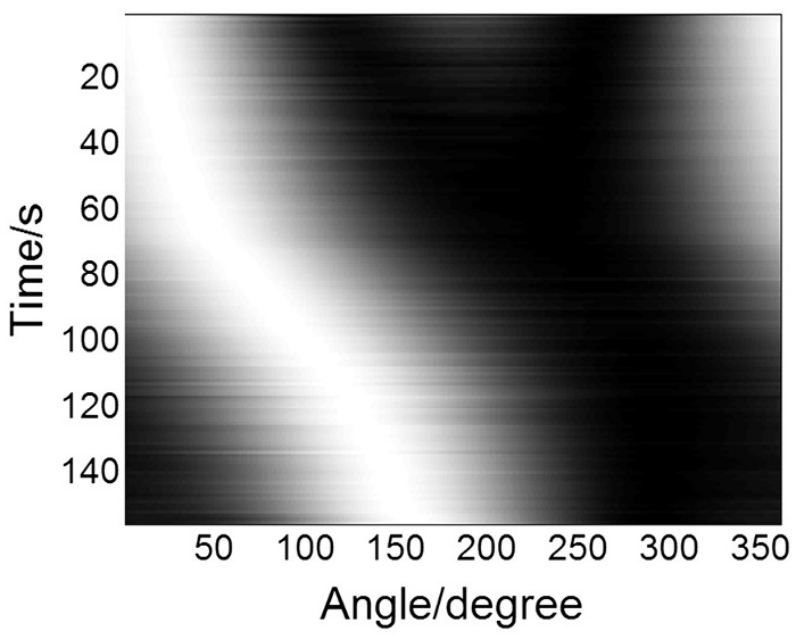

(b) Conventional beam-forming

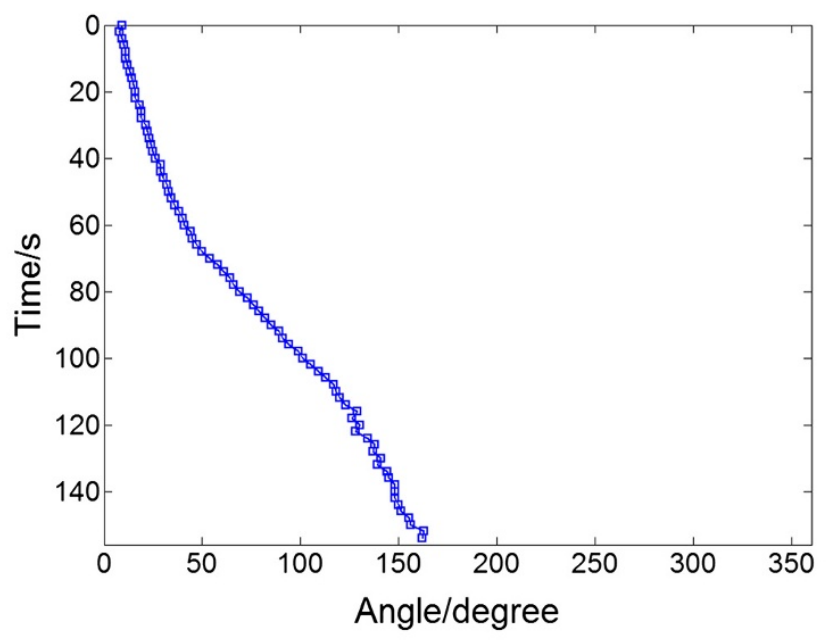

(c) Self-adapting root-MUSIC algorithm

Figure 6 Time-bearing display of motor boat. 


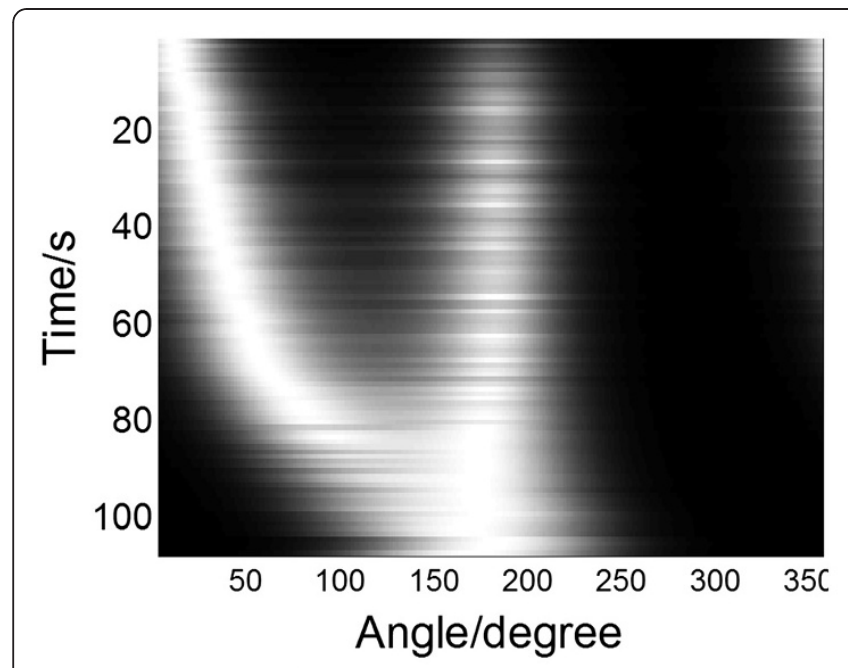

(a) MUSIC algorithm

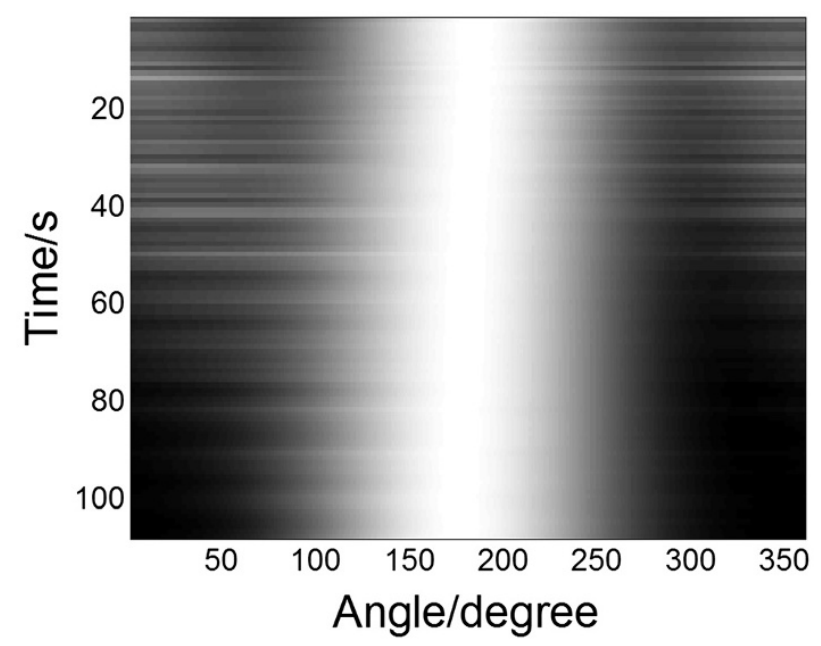

(b) Conventional beam-forming

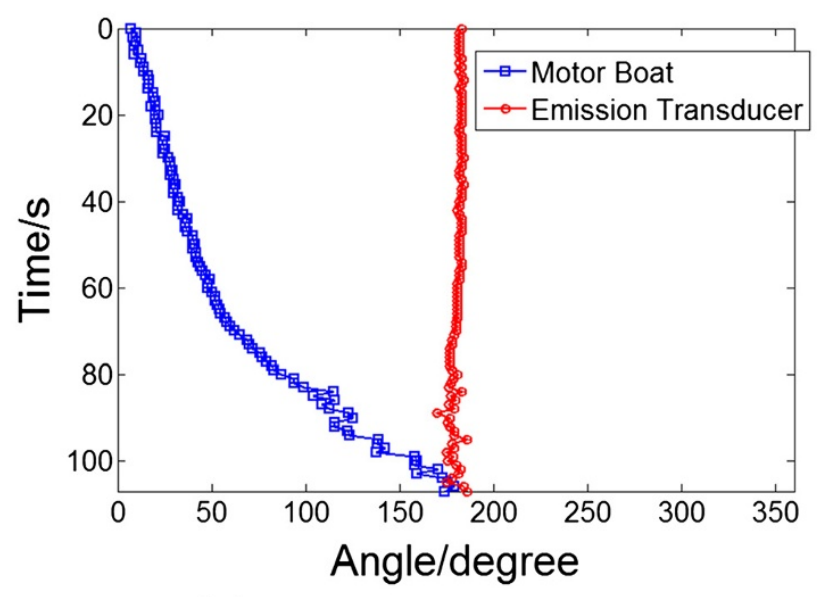

(c) RV-Root-MUSIC algorithm

Figure 7 Time-bearing display of motor boat and emission transducer.

\section{Conclusions}

The results of simulation experiment show the higher DOA estimation accuracy and lower RMSE of the new self-adapting root-MUSIC algorithm and the RV-RootMUSIC algorithm than the traditional MUSIC algorithm, and the results in lake trails show the engineering practicability of two new algorithms, it can be verified that the performance of RV-Root-MUSIC algorithm distinguishing the coherent signals.

\section{Competing interests}

The authors declare that they have no competing interests.

\section{Acknowledgements}

This work is supported by the National Nature Science Foundation of China (Grant No. 61127008) and International Science \& Technology Cooperation Program of China (Grant No.2010DFB10480).

\section{Author details}

${ }^{1}$ Key Laboratory of Instrumentation Science \& Dynamic Measurement, North University of China, Taiyuan 030051, China. ${ }^{2}$ Science and Technology on Electronic Test \& Measurement Laboratory, North University of China, Taiyuan 030051, China. ${ }^{3}$ School of Science, North University of China, Taiyuan 030051, China.

Received: 17 February 2012 Accepted: 10 October 2012

Published: 25 October 2012

\section{References}

1. C.B. Lesie, J.M. Kendall, J.L. Jones, Hydrophone for measuring particle velocity. J Acoust Soc Am 28, 711-715 (1956)

2. M.J. Berliner, J.F. Lindberg, Acoustic Particle Velocity Sensors: Design, Performance and Applications, American Institute of Physics (Woodbury, New York, 1996)

3. G.Q. Sun, Q.H. Li, Progress of study on acoustic vector sensor. ACTA ACUSTICA 29(6), 481-490 (2004). in Chinese

4. A. Nehorai, E. Paldi, Acoustic vector sensor array processing IEEE Trans. Signal Process 42(9), 2481-2491 (1994) 
5. P. Stoica, A. Nehorai, MUSIC, maximum likelihood and Cramer-Rao bound, IEEE Trans. Acoust. Speech, Signal Process 37(5), 2296-2299 (1989)

6. M. Hawkes, A. Nehorai, Acoustic Vector-Sensor Beamforming and Capon Direction Estimation. IEEE Trans. Signal Process. 46, 2291-2304 (1998)

7. M.D. Zoltowski, G.M. Kautz, S.D. Silverstein, Beamspace root-MUSIC. IEEE Trans Signal Process 41(1), 344-364 (1993)

8. B.D. Rao, K.V.S. Hari, Performance analysis of root-MUSIC. IEEE Trans. Acoust. Speech, Signal Process 37(12), 1939-1949 (1989)

9. C.Y. Xue, Z.M. Tong, B.Z. Zhang, A novel vector hydrophone based on the piezoresistive effect of resonant tunneling diode. IEEE Sensors Journal 8(4), 401-402 (2008)

10. C.Y. Xue, S. Chen, W.D. Zhang, Design, fabrication and preliminary characterization of a novel MEMS bionic vector hydrophone. Microelectronics Journal 38, 1021-1026 (2007)

11. T.D. Wen, L.P. Xu, J.J. Xiong, W.D. Zhang, The meso-piezo-resistive effects in MEMS/NEMS. Solid State Phenomena, 121-123 (2007). 619-622

12. W.D. Zhang, C.Y. Xue, J.J. Xiong, Piezoresistive effects of resonant tunneling structure for application in micro-sensors. Indian Journal of Pure \& Applied Physics 45, 294-298 (2007)

13. N. Jiang, J.G. Huang, S. Li, DOA estimation algortithm based on single acoustic vector sensor. Chinese Journal of Scientific Instrument 25(4), 87-90 (2004). in Chinese

14. M. Pesavento, A.B. Gershman, M. Haardt, Unitary Root-MUSIC with a Real-Valued Eigendecomposition: A Theoretical and Experimental Performance Study. IEEE Trans. Signal Process 48(5), 1306-1314 (2000)

15. D.A. Linebarger, R.D. DeGroat, E.M. Dowling, Efficient Direction-Finding Methods Employing Forward/Backward Averaging. IEEE Trans. Signal Process 42(8), 2136-2145 (1994)

doi:10.1186/1687-6180-2012-228

Cite this article as: Wang et al: Self-adapting root-MUSIC algorithm and its real-valued formulation for acoustic vector sensor array. EURASIP Journal on Advances in Signal Processing 2012 2012:228.

\section{Submit your manuscript to a SpringerOpen ${ }^{\circ}$ journal and benefit from:}

- Convenient online submission

- Rigorous peer review

- Immediate publication on acceptance

- Open access: articles freely available online

- High visibility within the field

- Retaining the copyright to your article 